\title{
Second order QCD corrections to gluonic jet production at hadron colliders
}

\author{
James Currie \\ Department of Physics, University of Zürich, Winterthurerstrasse 190, 8057 Zürich, Switzerland \\ E-mail: jcurrie@physik.uzh.ch
}

\section{Aude Gehrmann-De Ridder}

Institute for Theoretical Physics, ETH Zürich, 8093 Zürich, Switzerland

and

Department of Physics, University of Zürich, Winterthurerstrasse 190, 8057 Zürich, Switzerland

E-mail: gehraephys.ethz.ch

\section{Thomas Gehrmann}

Department of Physics, University of Zürich, Winterthurerstrasse 190, 8057 Zürich, Switzerland E-mail: thomas.gehrmann@uzh.ch

\section{Nigel Glover*}

Institute for Particle Physics Phenomenology, University of Durham, South Road, Durham DH1 3LE, England

E-mail: e.w.n.gloveredurham.ac.uk

\section{Joao Pires}

Dipartimento di Fisica, Universita di Milano \& INFN, Sezione di Milano, Via Celoria 16, Italy and Dipartimento di Fisica, Universita di Milano-Bicocca \& INFN, Sezione di Milano-Bicocca, Piazza della Scienza 3, 20126 Milan, Italy

E-mail: joao.pires@mib.infn.it

\section{Steven Wells}

Institute for Particle Physics Phenomenology, University of Durham, South Road, Durham DHI 3LE, England

E-mail: steven.wells@durham.ac.uk

\begin{abstract}
We report on the calculation of the next-to-next-to-leading order (NNLO) QCD corrections to the production of two gluonic jets at hadron colliders. In previous work, we discussed gluonic dijet production in the gluon-gluon channel. Here, for the first time, we update our numerical results to include the leading colour contribution to the production of two gluonic jets via quark-antiquark scattering.
\end{abstract}

Loops and Legs in Quantum Field Theory - LL 2014,

27 April - 2 May 2014

Weimar, Germany

* Speaker. 


\section{Introduction}

In hadron colliders, the production of high transverse momentum jets is the footprint of fundamental QCD processes. Single inclusive jet and dijet observables probe the basic parton-parton scattering in $2 \rightarrow 2$ kinematics. Precise measurements of these observables enables the determination of both the parton distribution functions in the proton and the strong coupling constant $\alpha_{s}$ up to the highest energy scales that can be attained in collider experiments.

Precision measurements of single jet and dijet cross sections have been performed at the Tevatron [1,2] and at the LHC operating at $\sqrt{s}=7 \mathrm{TeV}[3,4]$ and $\sqrt{s}=8 \mathrm{TeV}[5]$. The jet data are frequently included in global fits of parton distributions, where they provide crucial information on the gluon content of the proton and have been used to determinate the strong coupling by D0 [6] and CMS [7].

In QCD, the (renormalised and mass factorised) inclusive cross section for a dijet production in proton-proton collisions has the factorised form,

$$
\mathrm{d} \sigma=\sum_{i, j} \int \frac{d \xi_{1}}{\xi_{1}} \frac{d \xi_{2}}{\xi_{2}} f_{i}\left(\xi_{1}, \mu_{F}^{2}\right) f_{j}\left(\xi_{2}, \mu_{F}^{2}\right) \mathrm{d} \hat{\sigma}_{i j}\left(\alpha_{s}\left(\mu_{R}\right), \mu_{R}, \mu_{F}\right)
$$

where the probability of finding a parton of type $i$ in the proton, carrying a momentum fraction $\xi$, is described by the parton distribution function $f_{i}\left(\xi, \mu_{F}^{2}\right) d \xi$ and the partonic cross section d $\hat{\sigma}_{i j}$ for parton $i$ to scatter off parton $j$, normalised to the hadron-hadron flux ${ }^{1}$ is summed over the possible parton types $i$ and $j$. As usual $\mu_{R}$ and $\mu_{F}$ are the renormalisation and factorisation scales which are frequently set to be equal for simplicity, $\mu_{R}=\mu_{F}=\mu$.

For suitably high centre of mass scattering energies, the infrared-finite partonic cross section has the perturbative expansion

$$
\mathrm{d} \hat{\sigma}_{i j}=\mathrm{d} \hat{\sigma}_{i j}^{L O}+\left(\frac{\alpha_{s}\left(\mu_{R}\right)}{2 \pi}\right) \mathrm{d} \hat{\sigma}_{i j}^{N L O}+\left(\frac{\alpha_{s}\left(\mu_{R}\right)}{2 \pi}\right)^{2} \mathrm{~d} \hat{\sigma}_{i j}^{N N L O}+\mathscr{O}\left(\alpha_{s}^{3}\right)
$$

where the next-to-leading order (NLO) and next-to-next-to-leading order (NNLO) strong corrections are identified. The leading-order dijet cross section is proportional to $\alpha_{s}^{2}$.

Theoretical predictions for dijet observables are available to next-to-leading order (NLO) in QCD [8] and the electroweak theory [9]. The estimated uncertainty from missing higher order corrections on the NLO QCD predictions is substantially larger than the experimental errors on single jet and dijet data, and is thus the dominant source of error in the determination of $\alpha_{s}$. A consistent inclusion of jet data in global fits of parton distributions is currently only feasible at NLO. These theoretical limitations to precision phenomenology, coupled with the spectacular performance of the LHC and LHC experiments, means that next-to-next-to-leading order (NNLO) accuracy for dijet production is mandatory.

Jets in hadronic collisions can be produced through a variety of different partonic subprocesses. The $g g$ channel dominates at the LHC at low $p_{T}$ whereas at high $p_{T}$ the dominant processes are $q q$ and $q g$ scattering. The $q g$ channel has a contribution between $40-50 \%$ across the whole $p_{T}$

\footnotetext{
${ }^{1}$ The partonic cross section normalised to the parton-parton flux is obtained by absorbing the inverse factors of $\xi_{1}$ and $\xi_{2}$ into d $\hat{\sigma}_{i j}$.
} 

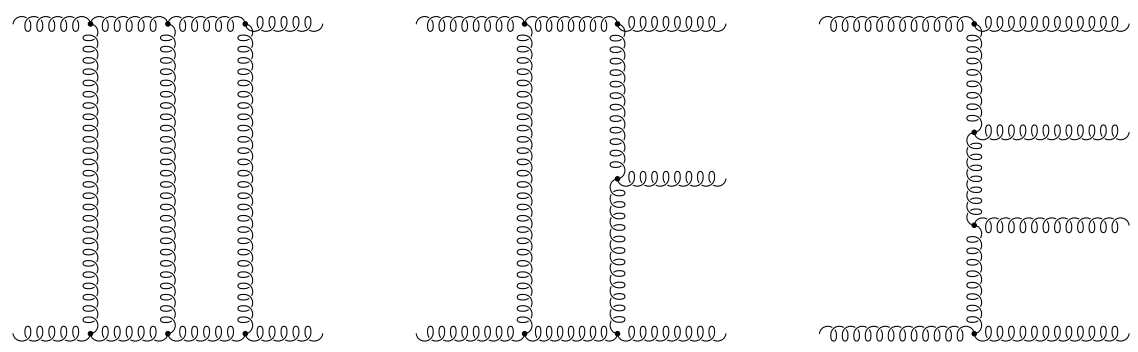

(a)
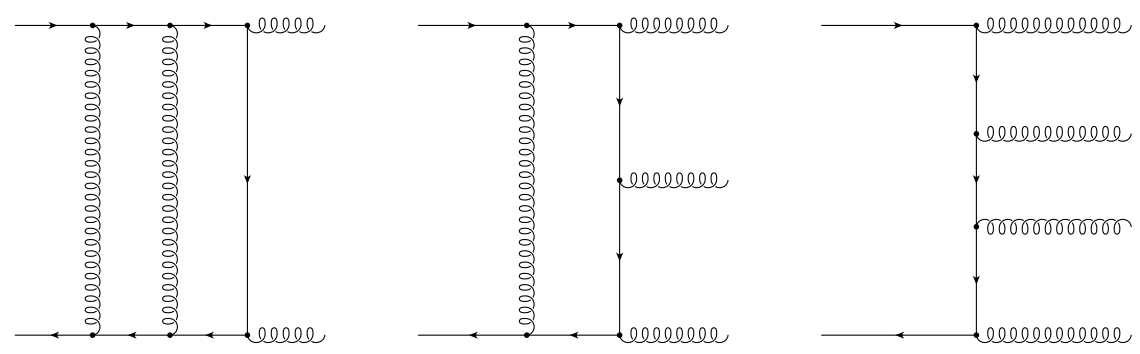

(b)

Figure 1: Representative Feynman diagrams at NNLO for (a) $g g \rightarrow$ gluons and (b) $q \bar{q} \rightarrow$ gluons.

range making it the second most dominant channel at the LHC. This is not the case at the Tevatron where $q g$ scattering is the dominant channel at low and moderate $p_{T}$ and the high- $p_{T}$ jet production is completely dominated by $q \bar{q}$ scattering. The first steps towards the NNLO corrections for this process were made in Refs. $[10,11]$ which computed the purely gluonic contribution to the dijet cross section, the $g g \rightarrow g g$ subprocess. In this contribution, we provide the first numerical results for the leading colour contribution to the $q \bar{q} \rightarrow g g$ subprocess. The NNLO calculation presented here describes gluonic jets production in the sense that only $g g \rightarrow$ gluons and $q \bar{q} \rightarrow$ gluons matrix elements are involved.

At NNLO, three types of parton-level processes contribute to jet production: the two-loop virtual corrections to the basic $2 \rightarrow 2$ process $[12,13]$, the one-loop virtual corrections to the single real radiation $2 \rightarrow 3$ process $[14,15]$ and the double real radiation $2 \rightarrow 4$ process at tree-level [16]. Representative Feynman graphs relevant for gluonic dijet production are shown in Fig. 1.

\section{Antenna subtraction and the NNLOJET integrator}

It is well known that in QCD, both the virtual and real radiative corrections are peppered with IR singularities which conspire to mutually cancel to form the finite physical cross section. After ultraviolet renormalization, the virtual contributions contain explicit infrared singularities, which 
are compensated by infrared singularities from single or double real radiation. These become explicit only after integrating out the real radiation contributions over the phase space relevant to single jet or dijet production. This interplay with the jet definition complicates the extraction of infrared singularities from the real radiation process. It is typically done by subtracting an infrared approximation from the corresponding matrix elements. These infrared subtraction terms are sufficiently simple to be integrated analytically, such that they can be combined with the virtual contributions to obtain the cancellation of all infrared singularities. The development of subtraction methods for NNLO calculations is a very active field of research and there are several methods on the market: sector decomposition [17], antenna subtraction [18], $q_{T}$-subtraction [19] and sectorimproved residue subtraction [20] have all been applied successfully in the calculation of NNLO corrections to exclusive processes.

Here we utilise the antenna subtraction formalism $[18,21]$ that was developed for the construction of real radiation subtraction terms. It is based on antenna functions constructed from physical matrix elements $[18,22]$ that each encapsulate all of the infrared singular limits due to unresolved radiation between two hard radiator partons. At NNLO, antenna functions with up to two unresolved partons at tree-level and one unresolved parton at one-loop are required. For hadron collider observables, one [23] or both [24,25] radiator partons can be in the initial state.

Our parton-level integrator, NNLOJET, can compute any infrared-safe observable related to gluonic dijet final states. NNLOJET is based around three integration channels, each identified by the multiplicity of the final state:

$$
\begin{aligned}
\mathrm{d} \hat{\sigma}_{i j, N N L O} & =\int_{\mathrm{d} \Phi_{4}}\left[\mathrm{~d} \hat{\sigma}_{i j, N N L O}^{R R}-\mathrm{d} \hat{\sigma}_{i j, N N L O}^{S}\right] \\
& +\int_{\mathrm{d} \Phi_{3}}\left[\mathrm{~d} \hat{\sigma}_{i j, N N L O}^{R V}-\mathrm{d} \hat{\sigma}_{i j, N N L O}^{T}\right] \\
& +\int_{\mathrm{d} \Phi_{2}}\left[\mathrm{~d} \hat{\sigma}_{i j, N N L O}^{V V}-\mathrm{d} \hat{\sigma}_{i j, N N L O}^{U}\right]
\end{aligned}
$$

For each choice of initial state partons $i$ and $j$, each of the square brackets is finite and well behaved in the infrared singular regions. For gluonic dijet production there are two channels, $g g \rightarrow$ jets and $q \bar{q} \rightarrow$ jets. The construction of the three subtraction terms $\mathrm{d} \hat{\sigma}_{g g, N N L O}^{S, T, U}$ was described at leading colour in Refs. [26, 27, 28] and at sub-leading colour in Ref. [11] while the leading colour subtraction terms for the $q \bar{q} \rightarrow$ gluons process were presented in Ref. [29]. It is a feature of the antenna subtraction method that the explicit $\varepsilon$-poles in the dimensional regularization parameter of one- and two-loop matrix elements are cancelled analytically against the $\varepsilon$-poles of the integrated antenna subtraction terms, while the implicit infrared poles present in the singular regions of the double-real and real-virtual phase space cancel numerically.

\section{Numerical results}

As in Refs. [10, 11], our numerical studies are based on proton-proton collisions at centre-ofmass energy $\sqrt{s}=8 \mathrm{TeV}$. We focus on the single jet inclusive cross section (where every identified jet in an event that passes the selection cuts contributes, such that a single event potentially enters the distributions multiple times) and the two-jet exclusive cross section (where events with exactly two identified jets contribute). 
Jets are identified using the anti- $k_{T}$ algorithm with resolution parameter $R=0.7$. Jets are accepted at central rapidity $|y|<4.4$, and ordered in transverse momentum. An event is retained if the leading jet has $p_{T 1}>80 \mathrm{GeV}$. For the dijet invariant mass distribution, a second jet must be observed with $p_{T 2}>60 \mathrm{GeV}$.

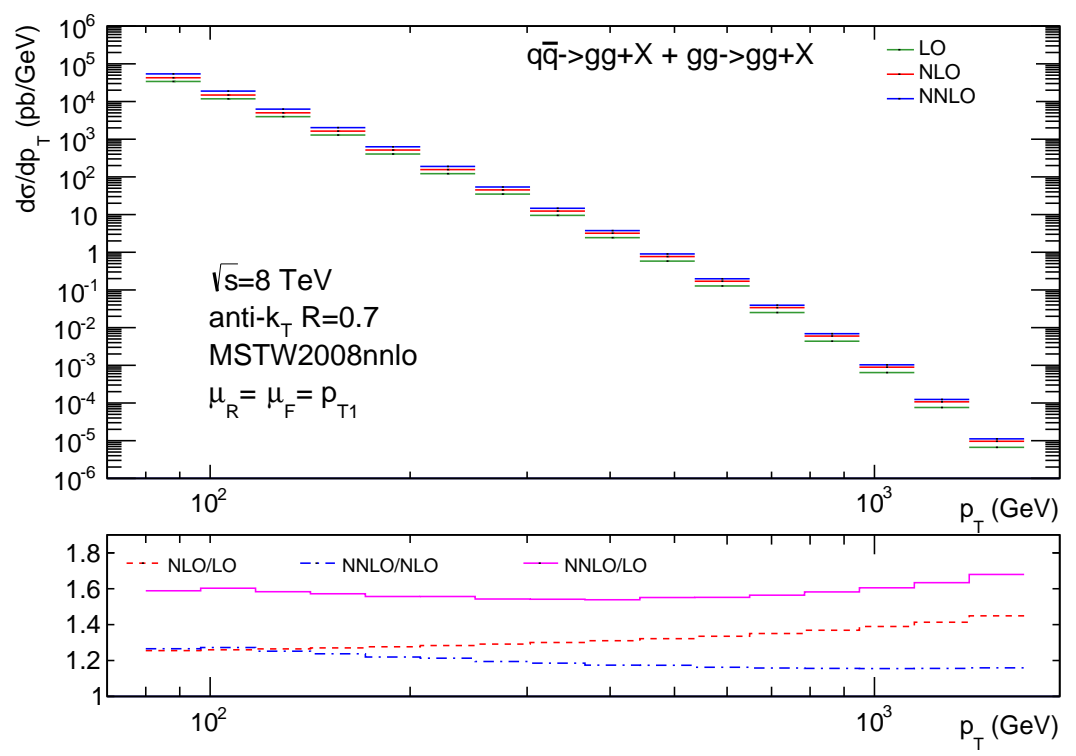

Figure 2: Inclusive jet transverse energy distribution, $d \sigma / d p_{T}$, for jets constructed with the anti- $k_{T}$ algorithm with $R=0.7$ and with $p_{T}>80 \mathrm{GeV},|y|<4.4$ and $\sqrt{s}=8 \mathrm{TeV}$ at NNLO (blue), NLO (red) and LO (dark-green). The lower panel shows the ratios of different perturbative orders, NLO/LO, NNLO/LO and NNLO/NLO.

All calculations have been carried out with the MSTW08NNLO distribution functions [30], including the evaluation of the LO and NLO contributions. This choice of parameters allows us to quantify the size of the genuine NNLO contributions to the parton-level subprocess. Factorization and renormalization scales $\left(\mu_{F}\right.$ and $\left.\mu_{R}\right)$ are chosen dynamically on an event-by-event basis. As default value, we set $\mu_{F}=\mu_{R} \equiv \mu$ and set $\mu$ equal to the transverse momentum of the leading jet so that $\mu=p_{T 1}$.

In Fig. 2 we present the inclusive jet cross section for the anti- $k_{T}$ algorithm with $R=0.7$ and with $p_{T}>80 \mathrm{GeV},|y|<4.4$ as a function of the jet $p_{T}$ at LO, NLO and NNLO, for the central scale choice $\mu=p_{T 1}$. The NNLO/NLO $k$-factor shows the size of the higher order NNLO effect to the cross section in each bin with respect to the NLO calculation. For this scale choice we see that the NNLO/NLO $k$-factor is approximately flat across the $p_{T}$ range corresponding to a $27-16 \%$ increase compared to the NLO cross section. Note that in the combination of $q \bar{q} \rightarrow g g+g g \rightarrow g g$ channels, the gluon-gluon initiated channel dominates. The NNLO/NLO $k$-factor for the $q \bar{q} \rightarrow g g$ channel alone is roughly $5 \%$.

Fig. 3(a) shows the inclusive jet cross section in double-differential form in jet $p_{T}$ and rapidity bins at NNLO. The $p_{T}$ range is divided into 16 jet- $p_{T}$ bins and seven rapidity intervals over the range 0.0-4.4 covering central and forward jets. The double-differential $k$-factors for the distribution in Fig. 3(a) for three rapidity slices: $|y|<0.3,0.3<|y|<0.8$ and $0.8<|y|<1.2$ are shown 


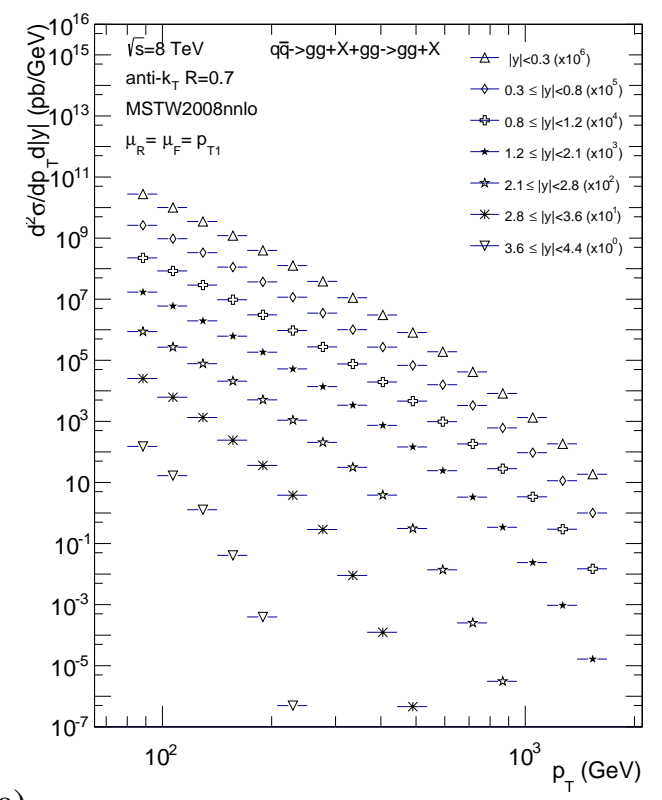

(a)

Figure 3: (a) The doubly differential inclusive jet transverse energy distribution, $d^{2} \sigma / d p_{T} d|y|$, at $\sqrt{s}=$ $8 \mathrm{TeV}$ for the anti- $k_{T}$ algorithm with $R=0.7$ and for $E_{T}>80 \mathrm{GeV}$ and various $|y|$ slices and (b) double differential $k$-factors for $p_{T}>80 \mathrm{GeV}$ and three $|y|$ slices: $|y|<0.3,0.3<|y|<0.8$ and $0.8<|y|<1.2$.

in Fig. 3(b). We observe that the NNLO correction increases the cross section between 27\% at low $p_{T}$ to $16 \%$ at high $p_{T}$ with respect to the NLO calculation (blue dot-dashed line) and this behaviour is similar for all three rapidity slices.

As a final observable, we computed the dijet cross section as a function of the dijet mass at NNLO. This is shown in Fig. 4 for the scale choice $\mu=p_{T 1}$ together with the LO and NLO results. The dijet mass is computed from the two jets with the highest $p_{T}$ and $\left|y_{1}\right|,\left|y_{2}\right|<4.4$ with $y^{*}$, defined as half the rapidity difference of the two leading jets $y^{*}=\left|y_{1}-y_{2}\right| / 2<0.5$. From Fig. 4(b), we see that the NNLO/NLO $k$-factor (blue dot-dashed line) increases the cross section between $25 \%$ at low $m_{j j}, 13 \%$ at moderate $m_{j j}$, to $20 \%$ at high $m_{j j}$. Once again this behaviour is similar for all three rapidity slices.

\section{Conclusions}

In conclusion, we have presented numerical results for the fully differential inclusive jet and dijet cross sections at hadron colliders at NNLO in the strong coupling constant using the partonlevel generator NNLOJET. We have considered the NNLO QCD corrections to the (full colour) $g g \rightarrow$ gluons and (leading colour) $q \bar{q} \rightarrow$ gluons subprocesses. The remaining contributions including the important $q g$ channel are in progress and will be reported on later. 


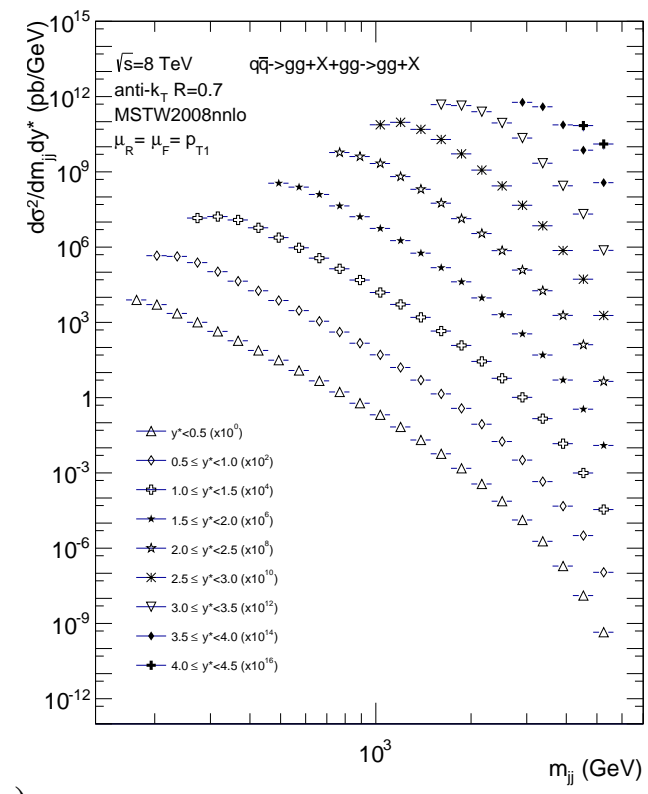

(a)

Figure 4: (a) Exclusive dijet invariant mass distribution, $d \sigma / d m_{j j} d y^{*}$, at $\sqrt{s}=8 \mathrm{TeV}$ for $y^{*}<0.5$ with $p_{T 1}>80 \mathrm{GeV}, p_{T 2}>60 \mathrm{GeV}$ and $\left|y_{1}\right|,\left|y_{2}\right|<4.4$ at NNLO (blue), NLO (red) and LO (dark-green) and (b) the ratios of different perturbative orders, NLO/LO, NNLO/LO and NNLO/NLO.

\section{Acknowledgements}

This research was supported in part by the Swiss National Science Foundation (SNF) under contract 200020-149517, in part by the UK Science and Technology Facilities Council as well as by the Research Executive Agency (REA) of the European Union under the Grant Agreements PITN-GA-2010-264564 (“LHCPhenoNet"), PITN-GA-2012-316704 ("HiggsTools"), and the ERC Advanced Grant MC@ NNLO (340983). JP acknowledges support by an Italian PRIN 2011 grant.

\section{References}

[1] CDF Collaboration, T. Aaltonen et. al., Phys. Rev. D78 (2008) 052006 [0807.2204].

[2] D0 Collaboration, V. M. Abazov et. al., Phys. Rev. Lett. 101 (2008) 062001 [0802.2400].

[3] ATLAS Collaboration, G. Aad et. al., Eur. Phys. J. C71 (2011) 1512 [1009.5908], Phys. Rev. D86 (2012) 014022 [1112.6297], JHEP 1405 (2014) 059 [1312.3524].

[4] CMS Collaboration, S. Chatrchyan et. al., Phys. Lett. B700 (2011) 187 [1104.1693], Phys. Rev. Lett. 107 (2011) 132001 [1106.0208], JHEP 1206 (2012) 036 [1202.0704], Phys. Rev. D 87 (2013) 11, 112002 [1212.6660].

[5] CMS Collaboration, S. Chatrchyan et. al., CMS-PAS-SMP-12-012, CMS-PAS-SMP-14-002.

[6] D0 Collaboration, V. M. Abazov et. al., Phys. Rev. D80 (2009) 111107 [0911.2710].

[7] CMS Collaboration, S. Chatrchyan et. al., CMS-PAS-SMP-12-028. 
[8] S. D. Ellis, Z. Kunszt and D. E. Soper, Phys. Rev. Lett. 69 (1992) 1496; W. T. Giele, E. W. N. Glover and D. A. Kosower, Phys. Rev. Lett. 73 (1994) 2019 [hep-ph/9403347]; Z. Nagy, Phys. Rev. Lett. 88 (2002) 122003 [hep-ph/0110315], Phys. Rev. D68 (2003) 094002 [hep-ph/0307268]; S. Alioli, K. Hamilton, P. Nason, C. Oleari and E. Re, JHEP 1104 (2011) 081 [1012.3380]; J. Gao et. al., Comput. Phys. Commun. 184 (2013) 1626 [1207.0513].

[9] S. Dittmaier, A. Huss and C. Speckner, JHEP 1211 (2012) 095 [1210.0438].

[10] A. Gehrmann-De Ridder, T. Gehrmann, E. W. N. Glover and J. Pires, Phys. Rev. Lett. 110 (2013) 16, 162003 [1301.7310].

[11] J. Currie, A. Gehrmann-De Ridder, E. W. N. Glover and J. Pires, JHEP 1401 (2014) 110 [1310.3993].

[12] E. W. N. Glover, C. Oleari and M. E. Tejeda-Yeomans, Nucl. Phys. B605 (2001) 467 [hep-ph/0102201]; E. W. N. Glover and M. Tejeda-Yeomans, JHEP 0105 (2001) 010 [hep-ph/0104178]; Z. Bern, A. De Freitas and L. J. Dixon, JHEP 0203 (2002) 018 [hep-ph/0201161].

[13] C. Anastasiou, E. W. N. Glover, C. Oleari and M. E. Tejeda-Yeomans, Nucl. Phys. B605 (2001) 486 [hep-ph/0101304]; Z. Bern, A. De Freitas and L. J. Dixon, JHEP 0306 (2003) 028 [hep-ph/0304168]; E. W. N. Glover and M. E. Tejeda-Yeomans, JHEP 0306 (2003) 033 [hep-ph/0304169].

[14] Z. Bern, L. J. Dixon, D. C. Dunbar and D. A. Kosower, Nucl. Phys. B425 (1994) 217 [hep-ph/9403226].

[15] Z. Bern, L. J. Dixon and D. A. Kosower, Nucl. Phys. B437 (1995) 259 [hep-ph/9409393].

[16] M. L. Mangano and S. J. Parke, Phys. Rept. 200 (1991) 301 [hep-th/0509223].

[17] T. Binoth and G. Heinrich,Nucl. Phys. $B 585$ (2000) 741 [hep-ph/0004013]; Nucl. Phys. B693 (2004) 134 [hep-ph/0402265]; C. Anastasiou, K. Melnikov and F. Petriello, Phys. Rev. D69 (2004) 076010 [hep-ph/0311311].

[18] A. Gehrmann-De Ridder, T. Gehrmann and E. W. N. Glover, JHEP 09 (2005) 056 [hep-ph/0505111].

[19] S. Catani and M. Grazzini, Phys. Rev. Lett. 98 (2007) 222002 [hep-ph/0703012].

[20] M. Czakon, Phys. Lett. B693 (2010) 259 [1005.0274]; Nucl. Phys. B849 (2011) 250 [1101.0642].

[21] A. Daleo, T. Gehrmann and D. Maitre, JHEP 0704 (2007) 016 [hep-ph/0612257].

[22] A. Gehrmann-De Ridder, T. Gehrmann and E. W. N. Glover, Phys. Lett. B 612 (2005) 49 [hep-ph/0502110], Phys. Lett. B 612 (2005) 36 [hep-ph/0501291].

[23] A. Daleo, A. Gehrmann-De Ridder, T. Gehrmann and G. Luisoni, JHEP 1001 (2010) 118 [0912.0374].

[24] R. Boughezal, A. Gehrmann-De Ridder and M. Ritzmann, JHEP 1102 (2011) 098 [1011.6631]; A. Gehrmann-De Ridder, T. Gehrmann and M. Ritzmann, JHEP 1210 (2012) 047 [1207.5779].

[25] T. Gehrmann and P. F. Monni, JHEP 1112 (2011) 049 [1107.4037].

[26] E. W. N. Glover and J. Pires, JHEP 1006 (2010) 096 [1003.2824].

[27] A. Gehrmann-De Ridder, E. W. N. Glover and J. Pires, JHEP 1202 (2012) 141 [1112.3613].

[28] A. Gehrmann-De Ridder, T. Gehrmann, E. W. N. Glover and J. Pires, JHEP 1302 (2013) 026 [1211.2710].

[29] J. Currie, E. W. N. Glover and S. Wells, JHEP 1304 (2013) 066 [1301.4693].

[30] A. D. Martin, W. J. Stirling, R. S. Thorne and G. Watt, Eur. Phys. J. C63 (2009) 189 [0901.0002]. 